\title{
Cerebral structure and intraventricular haemorrhage in the neonate: a real-time ultrasound study
}

\author{
MALCOLM I LEVENE, JONATHAN S WIGGLESWORTH, AND VICTOR DUBOWITZ
}

\author{
Department of Paediatrics and Neonatal Medicine, Institute of Child Health, Hammersmith Hospital
}

SUMMARY A method for systematic scanning of the neonatal brain with real-time ultrasound is described, and the linear array is compared with mechanical sector scanners. Anatomical landmarks recognised on axial, coronal, and parasagittal scans are verified by comparison with brain slices at necropsy. A prospective study of 50 infants admitted consecutively to the neonatal unit at this hospital showed intraventricular haemorrhage in $18(36 \%)$. These 18 infants included $10(43 \%)$ out of 23 of birthweight $\leqslant 1500 \mathrm{~g}$, and $3(27 \%)$ out of 11 of birthweight $1501-2000 \mathrm{~g}$. An unexpected feature was the recognition of intraventricular haemorrhage in 5 of the 13 infants $>2000 \mathrm{~g}$ birthweight. On sequential daily scans intraventricular haemorrhage was diagnosed most often in the first 2 days of life and abnormal ventricular echoes persisted for up to 12 days thereafter. Late development of hydrocephalus was recorded in 2 infants. Real-time ultrasound provides the neonatologist with a practical method for diagnosis and monitoring of intracranial lesions in the ill neonate and is a valuable, non-invasive, and safe tool for studying the pathophysiology of neurological handicap in infancy.

Intraventricular haemorrhage (IVH) is well known as a major cause of death in preterm infants. ${ }^{1-4}$ Prospective studies using computerised tomography (CT) have recently shown that IVH develops in 40 to $50 \%$ of infants of $\leqslant 1500 \mathrm{~g}$ birthweight, that traditional clinical criteria for diagnosis are unreliable, and that many infants survive this form of intracranial bleeding. ${ }^{5-7}$ This work implies that IVH is not merely a terminal event in ill neonates, but is a condition of major clinical importance which may make a significant contribution to the physical and mental handicap associated with preterm birth.

Accurate diagnosis of IVH during life has become of direct concern to the clinician. Although CT provides much new information on intracranial disease in the newborn infant, its value is limited by the need to send the infant to the CT unit, thus interfering with respiratory management and giving rise to the possibility of hypothermia. Since alterations in blood pressure and cerebral blood flow due to excessive handling may be important factors provoking IVH in susceptible infants, ${ }^{89}$ the procedure could indeed be a cause of the condition it seeks to demonstrate. The need for sedation and the high radiation dose are other disadvantages of routine or repeated use of CT scanning. It is thus essential to develop portable non-invasive techniques for diagnosis and monitoring of IVH and its sequelae.
Pape et al..$^{10}$ have reported their preliminary findings using B-mode real-time ultrasound with a linear array transducer for diagnosis of IVH and ventricular dilatation. Ultrasound is considerably attenuated by bone, but sufficient is transmitted through the poorly mineralised neonatal skull to provide good visualisation of intracranial structures.

We have examined various different ultrasound techniques for this purpose and report a systematic method for neonatal scanning. We describe the ultrasound findings using this technique in $\mathbf{5 0}$ infants admitted consecutively to the neonatal unit of this hospital.

\section{Methods}

Equipment. Ten commercially available real-time ultrasound machines have been evaluated in our neonatal unit. The instruments were of two different types: linear array and mechanical sector scanners. The linear array transducer consists of multiple piezo-electric crystals aligned in a row. The crystals are triggered to emit ultrasound pulses very rapidly in sequence, and returning echoes are detected and an image displayed on the screen. The mechanical sector scanner has a small number of piezo-electric crystals aligned on a wheel, or series of wheels, which rotate and emit an ultrasound pulse through a window at 
the base of the transducer. The machines that we found provided the best images and were the most convenient to use were the Kranzbuhler ADR with a 5 or $7 \mathrm{MHz}$ linear array transducer, the Toshiba Sonolayer-L SAL20A with a $3 \cdot 5 \mathrm{MHz}$ lineàr array transducer, and the ATL Mark III sector scanner
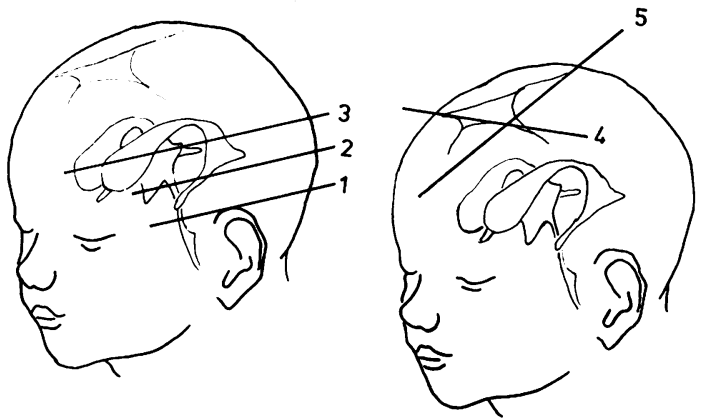

Fig. 1 Pattern of scanning for systematic examination of the neonatal head. with a $5 \mathrm{MHz}$ rotating wheel transducer. Vibration from the wheel which occurs in some mechanical scanners was not a problem with the ATL machine. In some cases a castor oil-filled bag attached to the linear array transducer was used to increase the area of acoustic contact, but we did not find it necessary to use this routinely. The ADR machine equipped with a $7 \mathrm{MHz}$ transducer gives excellent images through the fontanelle but does not penetrate bone.

Technique of scanning. The babies are scanned in three planes as shown in Fig. 1. Axial scans are performed using the brain stem as a landmark and starting at the canthohelical line. Two further scans are made at $1 \mathrm{~cm}$ intervals in planes above and parallel to this (lines 2 and 3 on Fig. 1). Coronal and parasagittal scans are performed using the anterior fontanelle as an acoustic window. Although the sections are described as distinct and separate, the transducer is moved over the head and intermediate
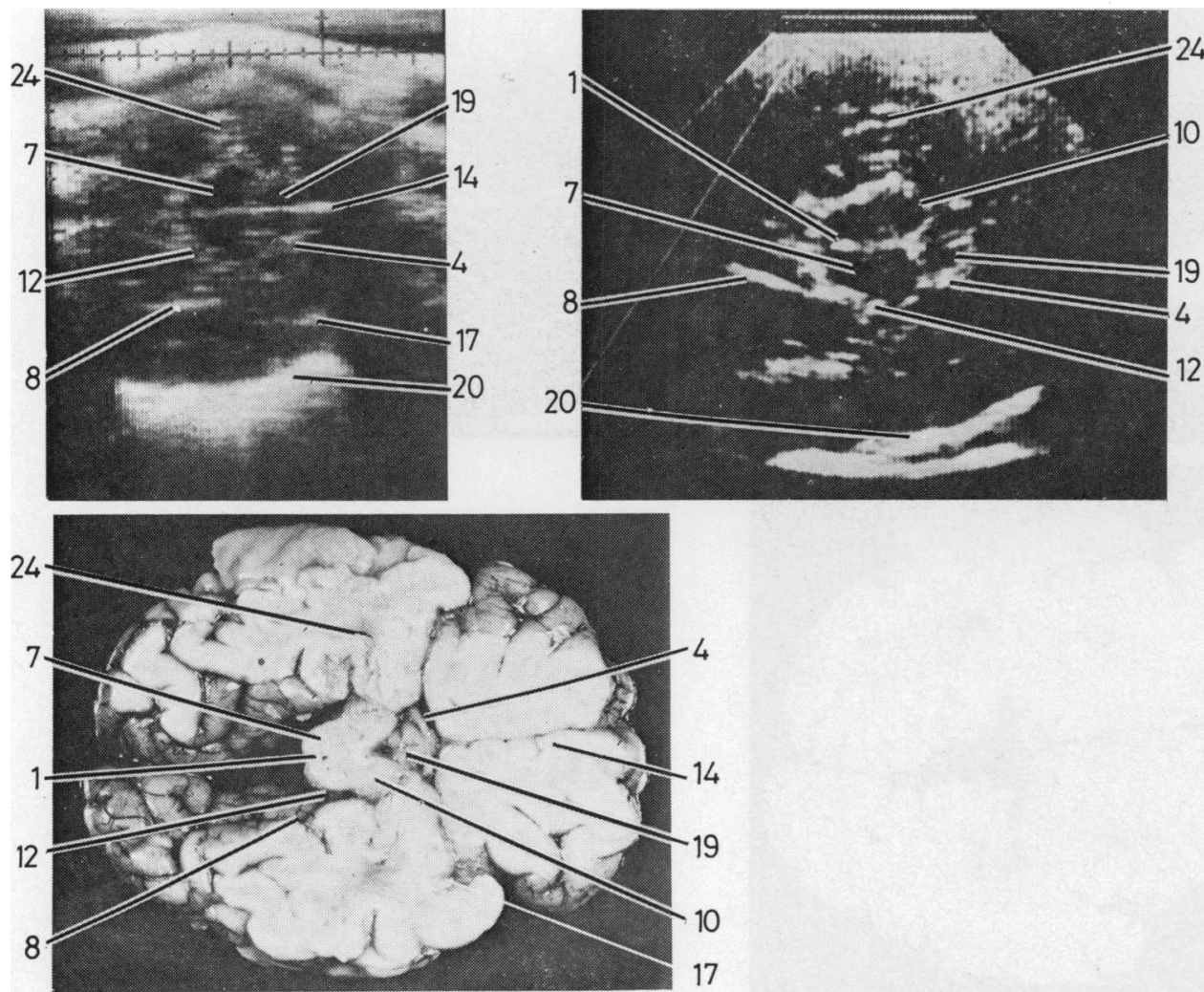

Fig. 2 Lowest axial section showing midbrain (7), anterior cerebral peduncles (10), aqueduct of Sylvius (1), mamillary bodies (19), internal carotid arteries (4), interhemispheric fissure (14), insula (17), calcarine fissure (8), vessels of the circle of Willis (12), inferior horn of lateral ventricle (24), and skull echo (20). 
anatomical features can be recognised. A full scan of the infant can be completed in the incubator in less than 5 minutes, with minimal handling of the baby.

Specimen ultrasound scans were compared with sections taken in the same plane and at the same level through brains of infants who had died without gross cerebral disease. The brains were fixed in $10 \%$ buffered formol saline for 2 weeks before sectioning. Some transducers used here produce a broad ultrasound beam of up to $1 \mathrm{~cm}$ wide, ${ }^{11}$ and echogenic structures within a centimetre of each other may appear superimposed on the screen as a composite image. For this reason two brain sections are shown with one ultrasound image in some examples. Fig. 7 shows an IVH on coronal section in an infant of 28 weeks' gestation. Necropsy permission was refused on this infant, but a coronal section of brain from an infant of similar gestational age with an extensive IVH is shown for comparison.
In Fig. 2 the lowest transverse axial scan, with the brain stem (7), and anterior cerebral peduncles (10) can clearly be seen. The aqueduct of Sylvius (1) is identified, and it may be seen to be dilated in communicating hydrocephalus. The internal carotid artery (4) and the vessels of the circle of Willis (12) can be clearly seen at this level and appear as pulsating images on the monitor screen. The inferior horn of the lateral ventricle may be identified in this plane (24).

Sections 2 and 3 (Figs 3 and 4) give information on the lateral ventricles (27). The interface between the cerebral hemispheres produces a strong echo (14) which is little diminished if the falx is removed during in vitro scanning of an excised brain. The interhemispheric fissure is seen as a composite image on scans 1, 2, and 3. The temporal branch of the middle cerebral artery (3) is seen just medial to the skull echo (20) in Fig. 4. The most anterior portion of
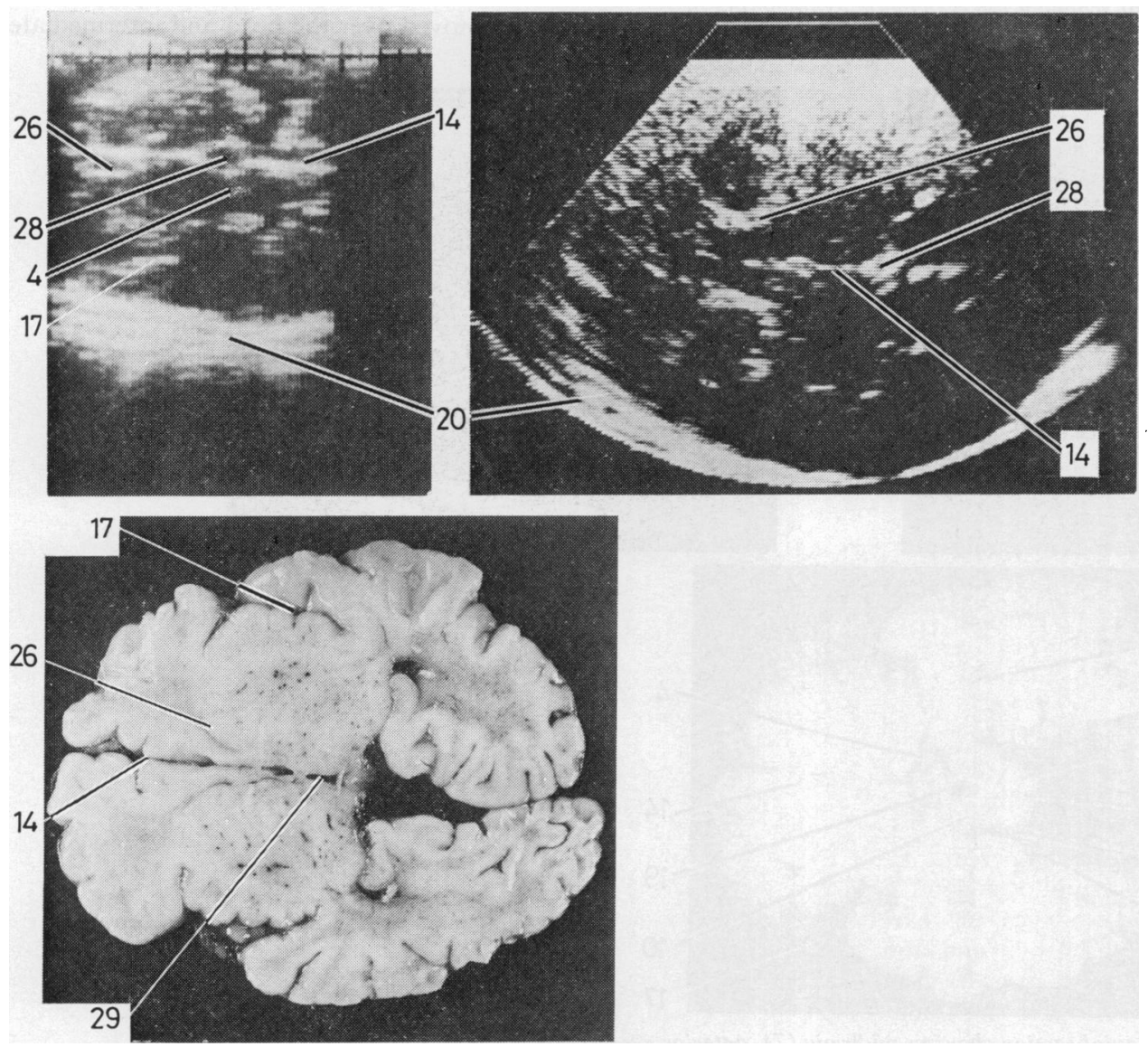

Fig. 3 Middle axial section through the cavum septi pellucidi (23), interhemispheric fissure (14), insula (17), anterior horn of lateral ventricle (26), internal carotid artery (4), and 3rd ventricle (29). 

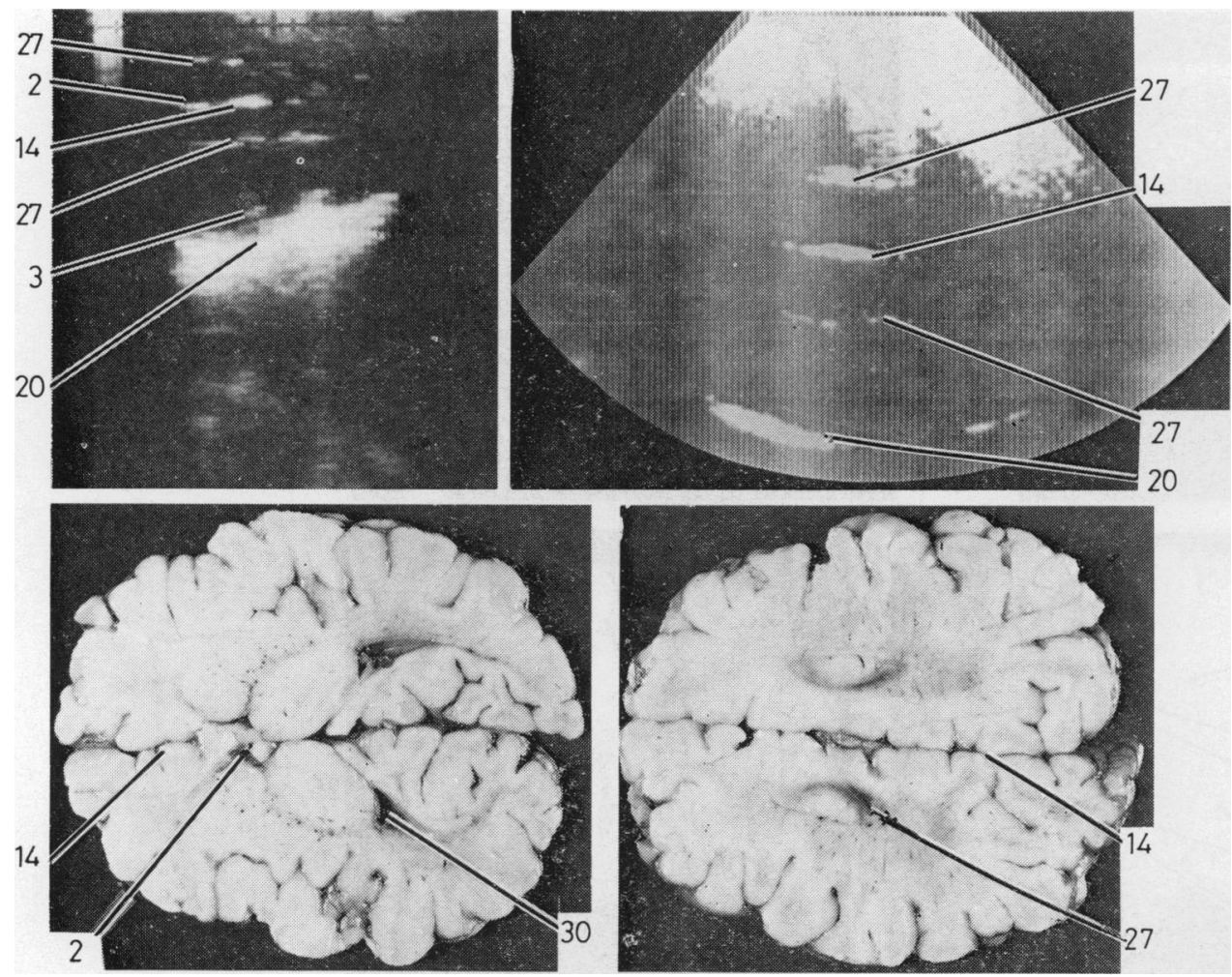

Fig. 4 Highest axial section through the lateral ventricle showing the interhemispharic fissure (14), lateral wail of the lateral ventricle (27), anterior cerebral artery (2), temporal branch of middle cerebral artery (3), occipital horn of the lateral ventricle (30), and the skull echo (20).

the lateral ventricle (26) is generally seen on the middle axial scan (Fig. 3) as well as the 3 rd ventricle (29) and septum pellucidum. The septum pellucidum is very often cystic in preterm infants and when present is considerably wider than the normal 3rd ventricle. We feel a midline echo-free cavity seen either in axial or coronal scans is likely to be a cavum septi pellucidi (28) rather than the normal 3rd ventricle.

The coronal section 4 (Fig. 5) clearly demonstrates the lateral ventricles (23). Due to poor contact of the linear array transducer with the vertex of the tightly curved neonatal head only a narrow section is imaged. The sector scanner due to its small transducer surface area, allows good contact with the fontanelle and produces an excellent image of the ventricles both normal and abnormal (25). The parasagittal section 5 (Fig. 6) visualises the lateral ventricles (23) well, and the thalamus (22) can be clearly seen. This plane offers a good view of the caudate nucleus (9) and germinal layer haemorrhages may be readily diagnosed. The cingulate sulcus (11) produces strong echoes and should not be confused with the roof of the lateral ventricle in this view. Haemorrhage (25) in the lateral ventricle gives the characteristic picture seen in Fig. 7.

\section{Results}

Using this method for intracranial scanning we prospectively examined 50 infants consecutively admitted to this hospital. These babies were all scanned on more than one occasion, and the total number of scans on any one baby ranged from 2 to 20. Most $(58 \%)$ of the infants had been born in the hospital, and the rest had been transferred from other hospitals. Whenever possible the first scan was performed within 24 hours of admission to our unit. Babies weighing less than $1500 \mathrm{~g}$, and babies requiring respiratory support generally had scans made daily for the first 2 weeks; thereafter scans were done weekly until discharge. Some of the infants discharged home have subsequently been rescanned in the follow-up clinic. Abnormal scans were 

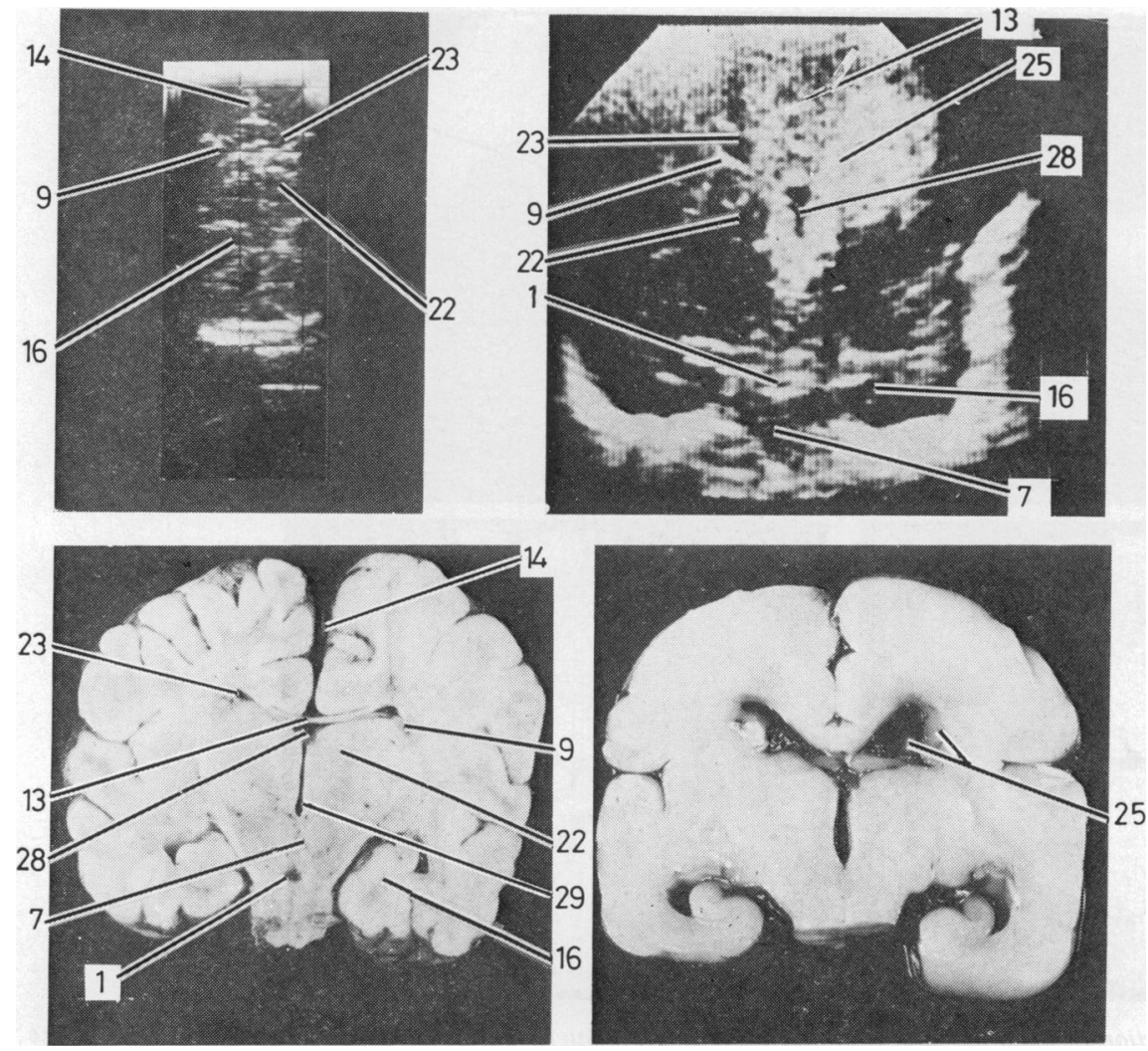

Fig. 5 Coronal section through the fontanelle showing the interhemispheric fissure (14), corpus callosum (13), normal lateral ventricle (23), caudate nucleus (9), hippocampus (16), aqueduct of Sylvius (1), brain stem (7), thalamus (22), and haemorrhage and oedema in the lateral ventricle (25).

Table 1 Incidence of abnormal and normal scans for various birthweight groups in 50 consecutive admissions to the neonatal unit

\begin{tabular}{|c|c|c|c|c|c|c|}
\hline & $\leq 1000 \mathrm{~g}$ & $1001-1500 \mathrm{~g}$ & $1501-2000 \mathrm{~g}$ & $2001-3000 \mathrm{~g}$ & $3001-4000 \mathrm{~g}$ & $>4000 \mathrm{~g}$ \\
\hline IVH (\%) & $3(50 \%)$ & $7(41 \%)$ & $3(27 \%)$ & $3(33 \%)$ & 2 & 0 \\
\hline Normal scan & 3 & 8 & 7 & 5 & 1 & 2 \\
\hline Hydrocephalus & $1^{*}$ & 1 & 0 & 0 & 1 & 0 \\
\hline
\end{tabular}

*This infant developed hydrocephalus after a documented IVH.

recorded with the time and date, and these data are summarised in Table 1.

Scans were described as either normal or as showing IVH, although no attempt was made at assessing the degree of severity. In 4 infants the scan was classified as being equivocal, as unclear or transient echoes were seen on one occasion. This probably represents a minor degree of bleeding and is shown as an IVH in Table 1. Table 2 shows the timing of IVH in infants in whom frequent and early scans
Table 2 Age at onset of IVH, and duration of time that echoes persisted in the lateral ventricles

\begin{tabular}{lllll}
\hline $\begin{array}{l}\text { Time of onset of } \\
\text { ventricular echoes }\end{array}$ & No of babies & $\begin{array}{l}\text { Duration } \\
\text { of echoes } \\
\text { (days) }\end{array}$ & Deaths & $\begin{array}{l}\text { No of infants } \\
\leqslant 1500 \mathrm{~g}\end{array}$ \\
\hline 24 hours & 5 & $1-7$ & 0 & 2 \\
$24-48$ hours & 6 & $1-12$ & 1 & 3 \\
$49-72$ hours & 1 & 3 & 1 & 0 \\
$4-6$ days & 1 & 8 & 0 & 0 \\
$7-10$ days & 3 & $1-7$ & 1 & 2 \\
\hline
\end{tabular}



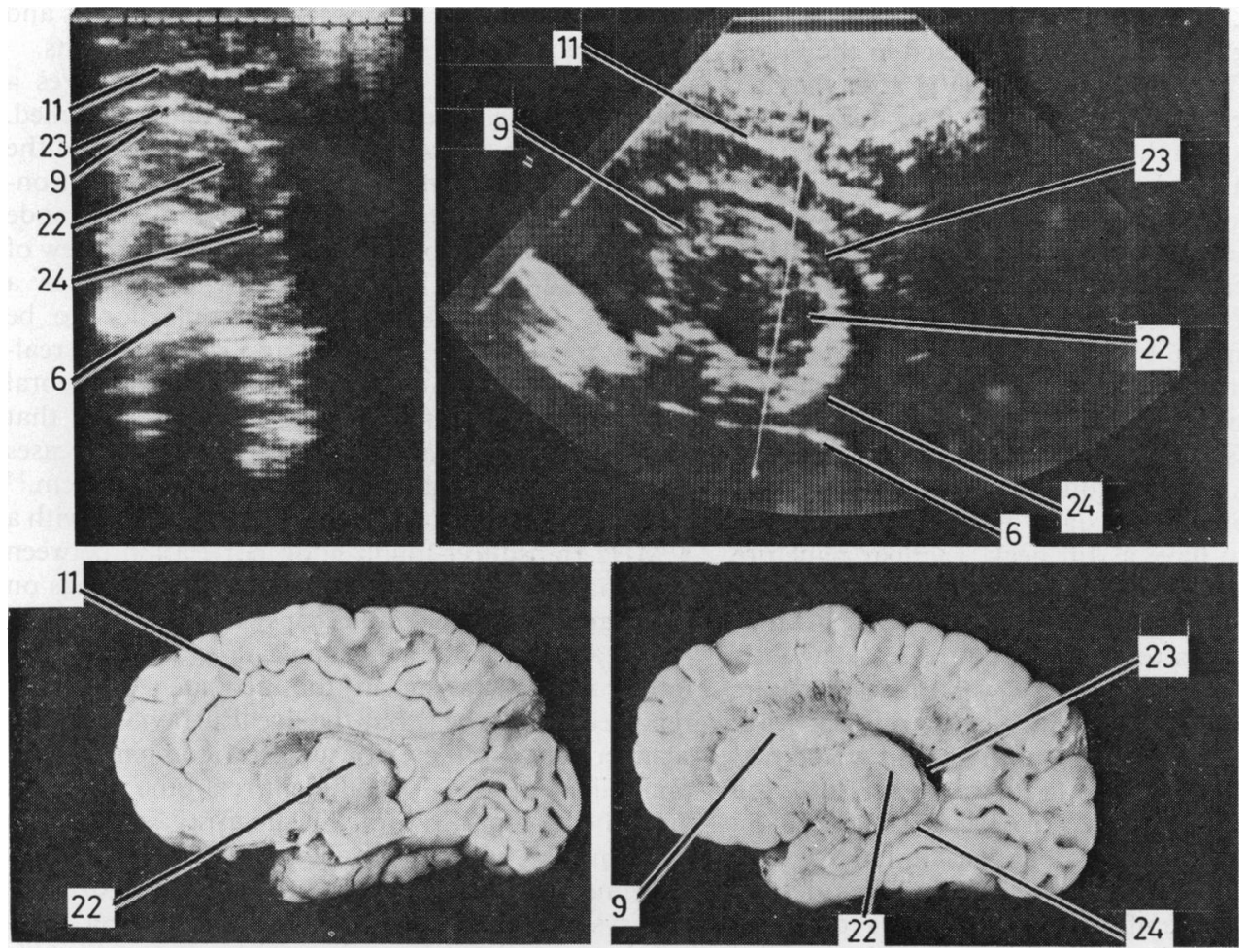

Fig. 6 A parasagittal section through the fontanelle showing the cingulate sulcus (11), lateral ventricle (23), caudate nucleus (9), thalamus (22), inferior horn of lateral ventricle (24), base of middle fossa (6).

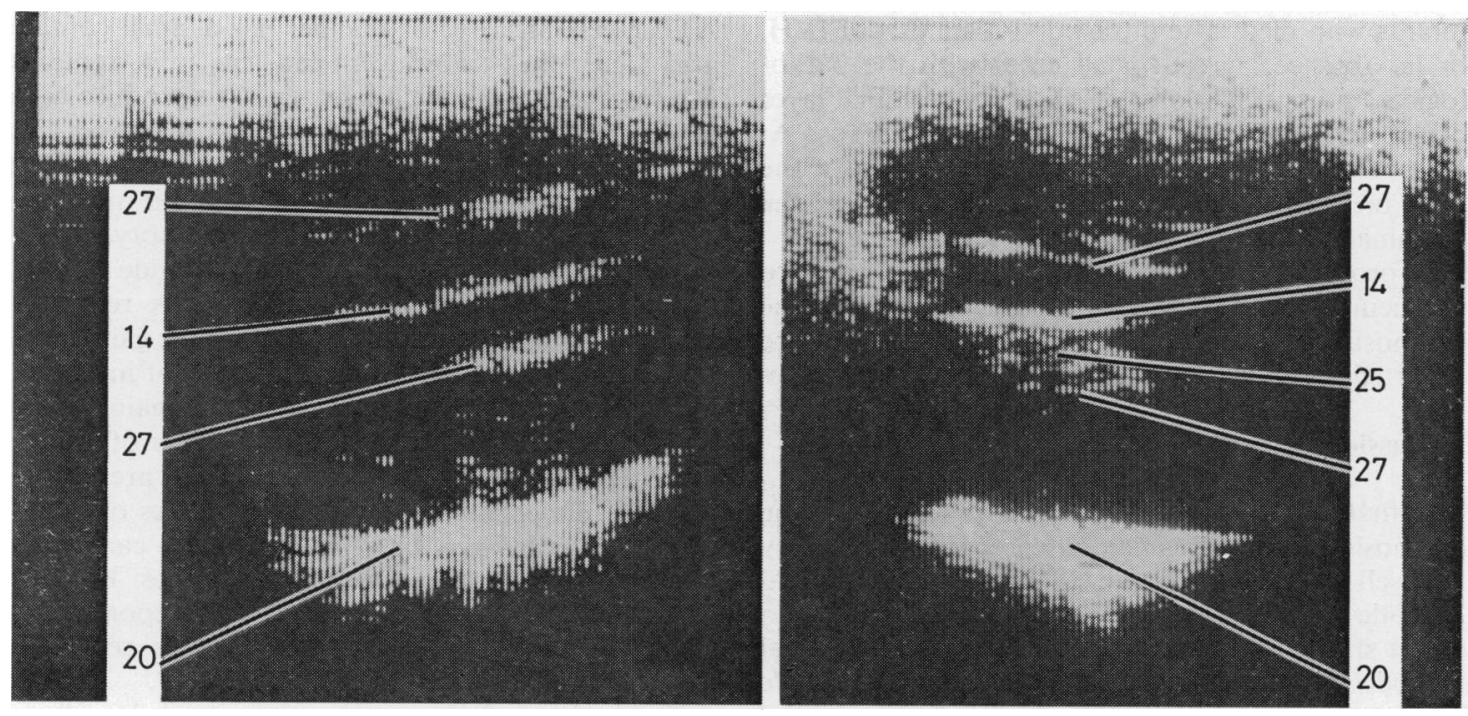

Fig. 7 Comparison of normal high axial scan (left) with a scan showing IVH in the lateral ventricle (25). Note the falx (14), lateral wall of lateral ventricle (27), and skull echo (20). 
were possible. In 11 out of the 16 babies, echoes corresponding to an IVH were detected in the first 2 days of life. Three babies had an IVH after the first week, the latest being at 11 days. The duration of detectable blood in the lateral ventricles ranged from 1 to 12 (median 5) days.

Two infants developed hydrocephalus after initial scans had shown normal lateral ventricular size. Dilatation occurred 26 days after birth in one infant, and in the other arrested hydrocephalus was noted on follow-up scan at 6 weeks. In the latter case a term, apparently normal infant was scanned at 20 hours of age on the postnatal ward. No abnormality in the scan could be seen, nor was there any abnormality in the ventricles. Twenty-four hours later the infant became irritable, pyrexial, and was found on examination to have a stiff neck. Lumbar puncture showed sterile, heavily blood-stained cerebrospinal fluid which did not clear in successive tubes. At that time the ventricles remained echo-free. A diagnosis of subarachnoid haemorrhage was made, and one day later echoes were seen in the lateral ventricles. This was thought to be organising blood which had refluxed through the ventricular system into the lateral ventricles. At follow-up 6 weeks later the baby was developing normally, but was found to have considerably dilated lateral and 3rd ventricles, despite a normal head circumference. At 6 months follow-up the ventricles remained dilated presumably due to a post-haemorrhagic obstruction; however the baby is neurologically normal, and her head growth is following a normal centile line.

Data were obtained at necropsy in 5 of the 7 infants who died. The ultrasound diagnosis of IVH, or its absence, agreed in all cases with the pathological findings. Not all IVHs were of germinal layer origin as some arose from the choroid plexus. At present one can seldom recognise the origin of the IVH on ultrasound scan unless it arises from the germinal matrix.

A prospective study of the correlations between the neurological assessment of the infant and the diagnosis of IVH by real-time ultrasound will be reported separately.

\section{Discussion}

The first report of the use of ultrasound for the diagnosis of intracranial disease was made by Lecksell in $1956 .{ }^{12}$ In the 1960 s, one-dimensional A-mode became a widely-used diagnostic tool to detect shifts in midline structures. Two-dimensional B-mode scanning gives much more qualitative information, and in the relatively poorly mineralised neonatal skull visualisation of intracranial structure is possible. Lees et al. ${ }^{13}$ have used static B-mode ultrasonography for diagnosis of hydrocephalus and intracranial cysts in infants aged below 16 months.

Static compound B-mode ultrasound requires a gantry to which the transducer is rigidly attached. This limits the portability of the equipment, and the baby must be moved from its intensive care environment to the scanning room. Real-time B-mode imaging provides a portable two-dimensional view of tissue, and scans rapidly enough to produce a 'moving' image, allowing arterial pulsation to be clearly seen. The first clinical application of a realtime B-mode system compared the size of the cerebral ventricles in 20 infants aged $<1$ year with that measured on $\mathrm{CT}$, and showed that in $85 \%$ of cases the biventricular width differed by less than $0.5 \mathrm{~cm} .{ }^{14}$ Pape et al. ${ }^{10}$ using an ADR linear array system with a $5 \mathrm{MHz}$ transducer found good correlation between IVH diagnosed by ultrasound scans and findings on CT examinations and at necropsy in 10 cases.

The advantages of a mechanical sector scanner for intracranical imaging in the neonate have been reported by Cooke, ${ }^{15}$ but the linear array and sector scanner have not previously been compared. Our preliminary study of 10 different real-time machines and the comparison of linear array systems to mechanical sector scanners permit objective iudgement of the benefits and disadvantages of these two systems. The flat linear array transducer gives good contact against the fairly flat temporoparietal region of the infant's head. Over the more tightly curved juvertex the contact is confined to $20 \%$ or less of the transducer area, although this can be improved by the use of a contact oil cushion. The ATL mechanical sector scanner has a small contact area and the transducer can fit into 'acoustic windows'-such as the fontanelles-to produce high quality images in the coronal and sagittal planes.

In a few infants, particularly those with moulded heads, good contact may be difficult and it may take considerable time to produce a satisfactory image. Babies with abundant hair may also provide contact problems and plentiful coupling jelly is required. Echoes may be amplified by turning up the gain of the signal and this may give the impression of increased intracranial echoes. With experience the gain can be set to give comparable images in infants of widely differing sizes, and artefactual echoes are not a problem. Discrimination of small volumes of blood in the ventricles may be difficult as there can be no objective cut-off point for minimal echoes. For this reason some scans are inevitably reported as equivocal. We believe that these cases represent minor degrees of bleeding.

The safety of diagnostic ultrasound has been considered since its first introduction into clinical practice. There has been no indication that there is 
any biologically harmful effect from diagnostic pulsed ultrasound of the frequency and intensity used here. Ultrasound has been used in obstetrics for over 15 years and applied to the fetal head with no proved complications. An infant may be scanned in the incubator with the minimum disruption of nursing care, and virtually no disturbance to the baby. In the course of over 500 ultrasound examinations to date, only 2 infants had a complication temporally related to scanning. The first was a 28-week gestation neonate who developed an apnoeic spell during the procedure. He was rapidly and easily resuscitated. The timing of subarachnoid haemorrhage 24 hours after the initial scan in the second infant suggests an ultrasound-induced complication. We believe this is unlikely as subarachnoid haemorrhage is fairly common in such infants, ${ }^{16} 17$ and its occurrence is probably no more than coincidence.

The incidence of IVH in infants $\leqslant 1500 \mathrm{~g}$ birthweight in our series was $43 \%$. This is identical with that recorded by Burstein et al. ${ }^{5}$ using the CT scanner. Although the occurrence of IVH in infants $>1500 \mathrm{~g}$ birthweight in not unusual, and the condition has been reported sporadically at term, 1819 the recognition of IVH in 5 out of 13 infants above $2000 \mathrm{~g}$ birthweight was a surprising and unexpected finding. Few of these infants showed any abnormal neurological signs, and the condition would have remained unrecognised in the absence of ultrasound studies, as there was no indication to do a CT scan.

The germinal matrix is gradually remodelled during the last trimester and is seldom seen as the origin of IVH in infants $>2000 \mathrm{~g}$ birthweight examined at necropsy. A more likely site of origin in this group is the choroid plexus. ${ }^{16} 2021$ Other possibilities are retrograde flow of blood from a subarachnoid haemorrhage (which we noted in one infant of $1950 \mathrm{~g}$ birthweight), and secondary haemorrhage into an area of periventricular infarction. Further studies and follow-up of these infants will be of particular interest.

The ability to time the onset of IVH in life is essential for any advance in knowledge of the pathophysiology of this condition. CT scanning is inadequate for this purpose as it cannot be performed repeatedly. Other techniques which have been described $^{22} 23$ use clot analysis at necropsy and are thus not of practical clinical application. We were able to time the onset of IVH to within 24 hours in most instances by scanning daily; more-precise timing would be possible by intensive study of a group of infants.

One of the problems of neonatal neurology is the lack of clinical signs associated with development of cerebral lesions in newborn infants. This has allowed gross intracranial lesions to go undiagnosed in the neonatal period and may be responsible for much persisting confusion over the causes of cerebral palsy. The development of real-time ultrasound scanning gives neonatologists the opportunity to look inside the head of the newborn infant at will. Integration of this technique with other forms of neonatal intensive care monitoring and more sensitive and objective clinical assessment of neurological function in the neonate ${ }^{24}$ should provide a basis for major advances in knowledge of neonatal neurology.

\section{Addendum}

The total number of cases we have now studied sequentially (up to November 1980) has risen to 202, and the incidence of IVH recognised by real-time ultrasound is similar to that reported here. We are thus unable to confirm the very high incidence of germinal layer haemorrhage reported by Bejar et al..$^{25}$

We thank Dr Sidney Leeman for help and encouragement with this study, and Mr W Trubridge and the Department of Medical Jllustration, Royal Postgraduate Medical School, Hammersmith Hospital, for preparing the illustrations.

The Wellcome Trust provided financial support.

\section{References}

1 Leech R W, Kohnen P. Subependymal and intraventricular hemorrhages in the newborn. Am J Pathol 1974; 77: 465-75.

2 Cole V A, Durbin G M, Olaffson A, Reynolds E O R, Rivers R P A, Smith J F. Pathogenesis of intraventricular haemorrhage in newborn infants. Arch Dis Child 1974; 49: 722-8.

3 Reynolds E O R, Taghizadeh A. Improved prognosis of infants mechanically ventilated for hyaline membrane disease. Arch Dis Child 1974; 49: 505-15.

4 Wigglesworth J S, Keith I H, Girling D J, Slade S A. Hyaline membrane disease, alkali, and intraventricular haemorrhage. Arch Dis Child 1976; 51 : 755-62.

5 Papile L, Burstein J, Kostler $\mathbf{H}$. Incidence and evolution of subependymal and intraventricular hemorrhage: a study of infants with birthweights less than $1,500 \mathrm{gm}$ $J$ Pediatr 1978; 92: 529-34.

6 Burstein J, Papile L A, Burstein R. Intraventricular hemorrhage and hydrocephalus in premature newborns: a prospective study with CT. AJR $1979 ; 132$ : 631-5.

7 Krishnamoorthy K S, Shannon D C, DeLong G R, Todres I D, Davis $K$ R. Neurologic sequelae in the survivors of neonatal intraventricular hemorrhage. Pediatrics 1979; 64: 233-7.

8 Hambleton G, Wigglesworth J S. Origin of intraventricular haemorrhage in the preterm infant. Arch Dis Child 1976; 51 : 651-9.

9 Wigglesworth J S, Pape K E. An integrated model for haemorrhagic and ischaemic lesions in the newborn brain. Early Hum Dev 1978; 2: 179-99. 
10 Pape K E, Blackwell R J, Cusick G, et al. Ultrasound detection of brain damage in preterm infants. Lancet 1979 ; i: $1261-4$.

11 Crisp C W, ed. Evaluation of real-time ultrasonic scanners. First report. Health Equipment Information No 81. London: DHSS, 1979: 21-7.

12 Lecksell L. Echo-encephalography. I. Detection of intracranial complications following head injury. Acta Chir Scand 1956; 110: 301-5.

13 Lees R F, Harrison R B, Sims T L. Grey scale ultrasonography in the evaluation of hydrocephalus and associated abnormalities in infants. Am J Dis Child 1978; 132: 376-8.

14 Skolnick M L, Rosenbaum A E, Matzuk T, Guthkelch A N, Heinz E R. Detection of dilated cerebral ventricles in infants. Radiology 1979; 131: 447-51.

15 Cooke R W I. Letter: Ultrasound examination of neonatal heads. Lancet 1979; ii: 38.

16 Pape K E, Wigglesworth J S. Pathological anatomy of intraventricular haemorrhage. In: Haemorrhage, ischaemia, and the perinatal brain. Clinics in Developmental Medicine No 69-70. London: Spastics International, 1979: 129 .

17 Volpe J J. Neonatal intracranial hemorrhage: pathophysiology, neuropathology, and clinical features. Clin Perinatol 1977; 4: 77-102.

18 Palma P A, Miner M E, Morriss F H, Jr, Adcock E W, Denson S E. Intraventricular hemorrhage in the neonate born at term. Am J Dis Child 1979; 133: 941-4.
19 Cartwright G W, Culbertson K, Schreiner R L, Garg B P. Changes in clinical presentation of term infants with intracranial hemorrhage. Dev Med Child Neurol 1979; 21 : 730-7.

20 Larroche J C. Post-haemorrhagic hydrocephalus in infancy: anatomical study. Biol Neonate 1972; 20 : 287-99.

21 Friede $\mathrm{R}$ L. Developmental neuropathology. Vienna: Springer, 1975.

22 Tsiantos A, Victorin L, Relier J P, et al. Intracranial hemorrhage in the prematurely born infant. $J$ Pediatr 1974; 85: 854-9.

23 Emerson P, Fujimura $M$, Howat $P$, et al. Timing of intraventricular haemorrhage. Arch Dis Child 1977; 52: 183-7.

24 Dubowitz L M S, Dubowitz V, Palmer P, Verghote $M$. A new approach to the neurological assessment of the preterm and full-term newborn infant. Brain Dev 1980; 2: 3-14.

25 Bejar R, Curbelo V, Coen R W, Leopold G, James H, Gluck $L$. Diagnosis and follow-up of intraventricular and intracerebral hemorrhages by ultrasound studies of infant's brain through the fontanelles and sutures. Pediatrics 1980; 66: 661-73.

Correspondence to Dr M I Levene, Department of Paediatrics and Neonatal Medicine, Hammersmith Hospital, Du Cane Road, London W12 0HS.

Received 30 April 1980 